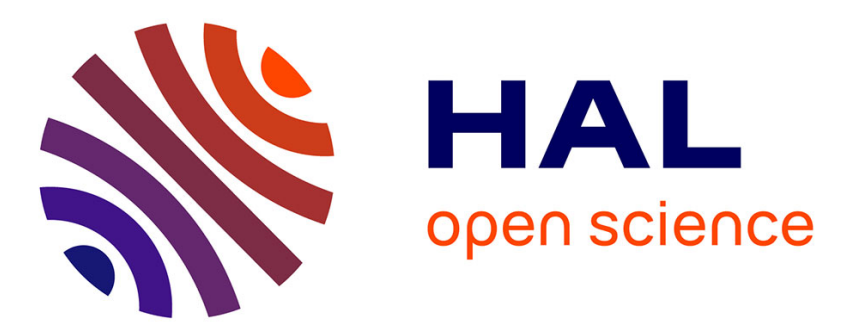

\title{
Thermal Signature Using Non-redundant Temporal Local Binary-Based Features
}

\author{
Adnan Al Alwani, Youssef Chahir, François Jouen
}

\section{To cite this version:}

Adnan Al Alwani, Youssef Chahir, François Jouen. Thermal Signature Using Non-redundant Temporal Local Binary-Based Features. International Conference on Image Analysis and Recognition, Jun 2015, Algarve, Portugal. pp.151-158. hal-01168439

\section{HAL Id: hal-01168439 \\ https://hal.science/hal-01168439}

Submitted on 25 Jun 2018

HAL is a multi-disciplinary open access archive for the deposit and dissemination of scientific research documents, whether they are published or not. The documents may come from teaching and research institutions in France or abroad, or from public or private research centers.
L'archive ouverte pluridisciplinaire HAL, est destinée au dépôt et à la diffusion de documents scientifiques de niveau recherche, publiés ou non, émanant des établissements d'enseignement et de recherche français ou étrangers, des laboratoires publics ou privés. 


\title{
Thermal Signature Using Non-Redundant Temporal Local Binary-based features
}

\author{
Adnan AL ALWANI ${ }^{1}$ Youssef CHAHIR ${ }^{1}$ \\ Francois JOUEN ${ }^{2}$ \\ ${ }^{1}$ GREYC CNRS (UMR 6072) \\ University of Caen Basse-Normandie \\ ${ }^{2}$ CHArt Laboratory \\ Practical School of High Studies (EPHE) Paris \\ ${ }^{1}$ \{adnan.alalwani, youssef.chahir\}@unicaen.fr \\ 2 francois.jouen@ephe.sorbonne.fr
}

\begin{abstract}
In this paper, we propose a method for events recognition from thermal signature based 1-D signal. We use the non-redundant temporal Local Binary Pattern NRTLBP as a descriptor of the Pattern Of Interest (POI) signal. The original signal is extracted directly from local patch in region of interest. We introduce the wavelet decomposition as a pre-processing stage in order to extract the approximation wave-components of the raw signal. Then, NRTLBP is applied on the wave-components which provide wavelet domain descriptor of the raw thermal signature. Finally, we provide an evaluation of our method on the real dataset (Preterm Pain in Infants "PPI") composed of thermal videos developed in the context of Infant pain project, a french project supported by the French National Research Agency Projects for science (ANR).
\end{abstract}

Keywords: Events recognition. Non-Redundant Temporal Local Binary Pattern. Thermal signature. Support Vector Machine, Wavelet

\section{Introduction}

Feature extraction is an important challenge in pattern recognition, specifically if the pattern is represented by a temporal or spatial signal such as speech, acoustic signal or $2 \mathrm{D}$ signal. The temporal signals have finite durations and are sampled into digital format. In such case, it is more appropriate to represent a pattern as a finite time sequence $\{x[0], x[1], \ldots, x[N-1]\}$. Providing this sequence directly to a classifier is not typical due to the huge number of inputs and due to the randomness of the signal. Therefore, the sequence $x[n]$ must be encoded into a low-dimension feature vector $Y=\left(y_{1}, y_{2}, \ldots, y_{D}\right), D<<N$, which better characterizes the pattern and provides a robust classifier training and classification. Feature extraction from One-Dimension (1-D) signal is generally categorized into two approaches : temporal and spectral approach. Both approaches are 
deemed as nonparametric approaches. As well as parametric approaches, both approaches have been used in classification of many patterns examples. Spectral approaches have gained a big popularity in the last two decades, which are out of scope of this paper. In contrast, in the temporal approach, the features are extracted directly from the temporal sequence. Moreover, use of multifunction allows to characterize the time sequence by the following quantities : mean value, slope absolute value, number of zero crossings, slope sign changes, and waveform length. Although there are techniques that claimed a successful discrimination in various applications, the above temporal characterizations didn't help much in the other applications. Techniques from the fields of machine learning and pattern recognition might be valuable for thermal pattern examination. Thermal pattern may have sufficient knowledge to understand the underlying characteristics of the patterns that we observe. However, reliable classification of thermal signature would help us to learn about an unknown physical aspects, discriminate the normal and abnormal states in the condition monitoring. The realization of such a tool can be difficult task in some cases.

This paper presents an approach for event recognition from temporal thermal signal. The recognition process is based on the Non-Redundant Temporal Local Binary Pattern-based features (NRTLBP) over the time series thermal sequences which are extracted from the thermal video sequences. A traditional way to characterize the properties of a time series sequence $x[n]$ is through its raw temperature value extracted from local patches that are defined over the objects face in image sequences. It has been shown that if a signal has band-limited characteristics, significant improvement in the performance of pattern recognition can be readily made by a relatively simple preprocessing of the signal in the time-frequency domain. The redundant representation of a $1 \mathrm{D}$ signal in a $2 \mathrm{D}$ time-frequency domain can provide an additional degree of freedom for signal analysis. Such pre-processing effectively separates the intertwined time domain features of the signal, allowing the important characteristics to be exposed in the time-frequency domain, resulting in more effective pattern matching. Hence, the signal being analyzed needs to be conducted only around in selected regions of interest in the time-frequency domain. For this, we use the wavelet transform to decompose the signal into pyramid structure in the form of approximation component and detail component. The approximation wave components are also known to be a robust and distortion tolerant feature space for many pattern recognition applications. Moreover, we consider the approximation component as the feature vector of raw signal, which is a necessary requirement for extracting NRTLBP-based features. As the temporal LBP produces efficient feature descriptor, we employ a Support vector Machine SVM to evaluate our method on the real Preterm dataset.

Extracting features from one-dimensional signal based on thermal video monitoring methods, have been considered in a number of approaches. The body work of these approaches is based on measurement, quantifying and tracking of thermal signature. Many other approaches in the field of the thermal signature exam- 
ination have been proposed in the literature. In [1] the authors examined the temperature changes around the neck and the nasal regions. These regions were identified manually in the recorded images, then the respiration rate signals were extracted from the thermal image by performing wavelet transform. Respiration signal rate monitoring is also explored in [2]. A tracking algorithm is developed to follow three facial features in order to produce the respiration signal rate. New non-contact respiration signal modality for neonatal was developed and investigated by [3], using infrared thermography imaging. This development includes subsequent image processing (region of interest (ROI) detection) and optimization based wavelet technique. In [4] F. AL-Khalidi et al. developed a technique to model the respiration signal from nasal region. A feature extracted from this region enabled a respiration signal to be produced. The ROI under the tip of the nose was segmented into eight parts. The pixel values within each segment were averaged to obtain a single value representing that segment. Additionally to the direction of vital signal examination. The approach in [5] used mid-wave IR sensors for the measurement of cardiac and breathing rates at a distance. They used the Fourier Transform to isolate the relevant frequency components from skin temperature in the vicinity of the major arteries. Furthermore, they showed that the frequency components from skin temperature is directly related to the pulse waveform related to the heart signal.

Our method differs from other approaches by taking the root of pattern recognition to recognize the event from thermal video. We investigate the discrimination power of NRTLBP in the field of the thermal pattern descriptor in Section 3, whereas Section 4 illustrates its performance using wavelet transform to decompose the raw statistical features and build new feature representation. In Section 5 , the experimental protocol is recalled and we conduct comprehensive experiments to demonstrate the effectiveness of our approach in recognizing if an infant is in pain. Finally, conclusions are drawn in Section 6.

\section{A Framework for Event Recognition}

We propose a framework of using thermal attributes extracted from the video sequences. We assume that the layout of subjects is considered for all the cases in the front view, and ROI is defined over the subjects face. In addition, we build raw thermal signatures for all subjects samples. This is achieved first by defining local patch as shown in the Fig1. Then the maximum $M x$ and the minimum $M n$ values are computed from the local patches along the video sequences. For more illustration, we compute the $M x$ and $M n$ temperature values and defined it as the raw thermal signals (we call it raw signals for abbreviation). Furthermore, we establish the raw signals for the three condition events, which characterize the monitoring state during daily care. For instance, normal event, disease event and post-disease event are three events adopted in our study. The time series of each event is shown in the Fig2. As illustrated in this figure, the response nature of each event specify the discriminative features of that event.

Then, we apply the NRTLBP descriptor on the raw thermal signal in order 
to extract efficient feature vector. Another extension consists to approximate components, before using NRTLB, via wavelet decomposition of the temporal raw signal. A video sequence is then described by two descriptors which are directly applied into SVM for event recognition.

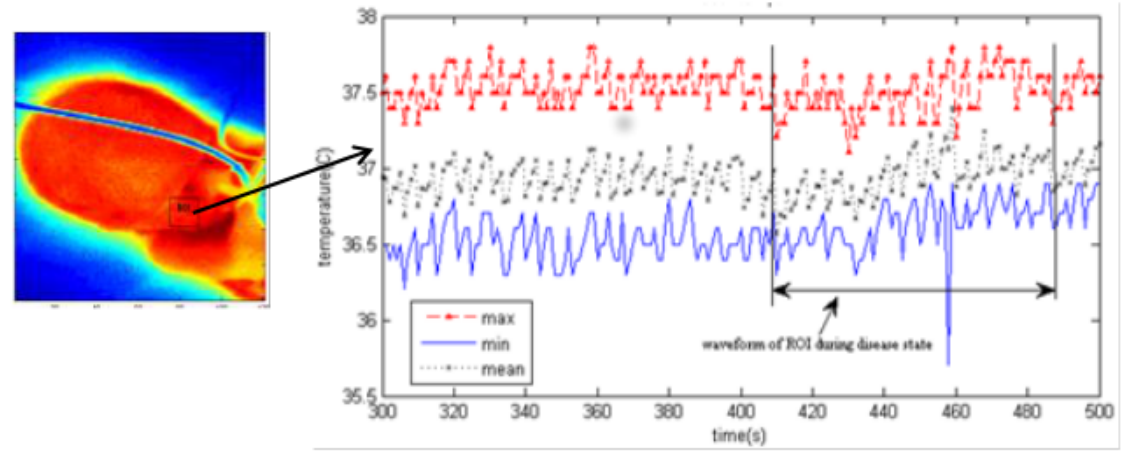

Fig. 1. Local temperature extracted from ROI defined over the object's face.

\section{Non-Redundant Temporal Local Binary Pattern-based features}

Once the thermal features are extracted, the video sequences is considered as a collection of raw signals. We denote by $X_{i}=T_{1}, T_{2}, \cdots, T_{n} . i=M x$ or $M n$, and $T$ is the temperature value at each patch. In order to represent the raw signal in efficient manner we propose the Temporal Local Binary Pattern descriptor, which is an extension of original 2-D LBP operator into the temporal domain. Normal LBP has originally been proposed for texture analysis and classification [6]. Recently, it has been applied on face recognition[7] and facial expression recognition[8]. The TLBP operator labels the samples of a signal by thresholding a center sample against neighborhood set within defined window. We denote by $x[n]$ the sampled signal, and $w$ is the size of samples window. The TLBP operator on a sample $k w$ in is given by:

$$
T L B P_{w}(f[k])=\sum_{q=0}^{\frac{w}{2}-1}\left(f[k]-f[k-j-q] 2^{q}+f\left[\frac{w}{2}+q+1\right]-f[k] 2^{q+\frac{w}{2}}\right.
$$

Where $q$ is the number of sampled points (neighbor samples of $\mathrm{k}$ ) whose the distances to $k$ do not exceed the window $w$. For a $M$ block, a TLBP histogram of 

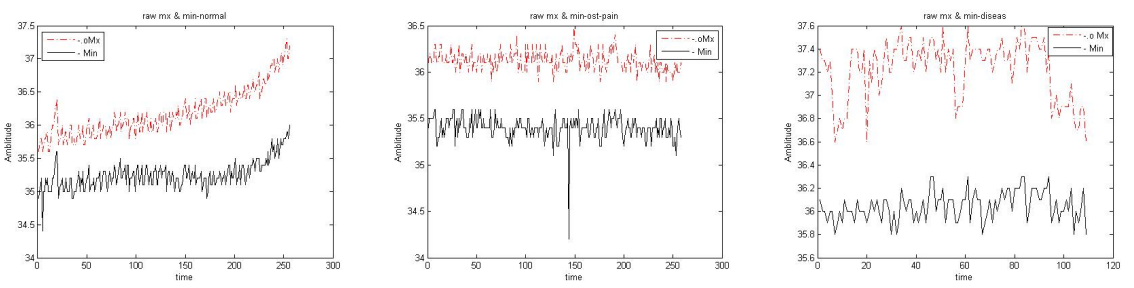

Fig. 2. Raw thermal signals of Mx and Min temperature values. From top to bottom, normal event, disease event and post-disease event signatures

$2^{q}$ bins is computed for feature representation. After the computation of the LBP for the whole signal, a histogram of the TLBP code is used as feature descriptor. It contains information about the distribution nature over the whole signal and characterizing a statistical description of signal. Another aspect of TLBP is called no-redundant TLBP. Intuitively, the NRTLBP considers a TLBP code and its complement as same. NRLBP is defined by:

$$
N R T L B P(f[k])=\min \left(T L B P(f[k]), 2^{q}-1-T L B P(f[k])\right)
$$

This is motivated by the advantage of using dynamic shape of the signal at various instances and levels. Finally, the no-redundant local patterns formed from signal $f[k]$ are formed as a histogram distribution vector.

\section{NRTLBP of Approximated Coefficients}

We further extend the idea of LBP into a wavelet domain in order to perform the comparison results. The method is based on using multi-resolution wavelet transform to decompose the raw statistical features and build new feature representation [9].

The wavelet transform $C(j, k)$ of a finite-energy signal $f(t)$ is defined as its scalar product with the wavelet $\Psi_{j k}(t)$. In other words, the wavelet transform represents the correlation of the signal $f(t)$ and the wavelet $\Psi_{j k}(t)$ as:

$$
a_{j k}=\sum_{t} f(t) \Psi_{j k}(t)
$$

Where $\Psi(t)=2^{j} \Psi\left(2^{j} t-k\right)$ is the mother wavelet corresponds to scale $j=1$, $k$ is the translation factor, and $j$ is the scale parameter. A wavelet representation of a function consists of, a coarse overall approximation $a^{j}$, and detail coefficients that influence the function at various scales. Therefore, the approximation coefficients are considered as raw feature vector in a wavelet-domain. Then using NRTLBP descriptor for feature description. The smooth change property of the approximation features is illustrated in the Fig 4 . Where each subfigure shows the feature in wavelet-domain for the corresponding event in the time domain explained in the Fig. 2. 

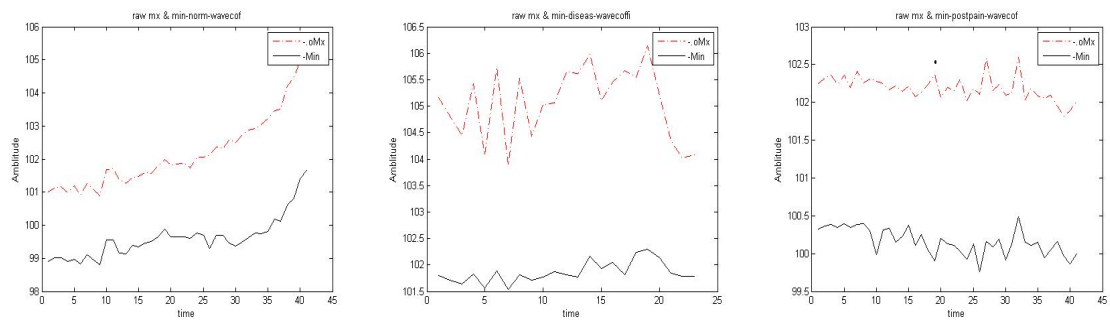

Fig. 3. Approximation component of raw features for three kinds of events. from top to bottom, normal event, disease event, and post-disease event signatures.

\section{Experiments}

In this section, we evaluate the proposed method by experimenting with the real dataset from ANR pretherm project. Due to non available of the benchmark related to our method, we did not attempt a comparison with other methods. Rather, we provide results only to show that our algorithm works well on a real data. The preterm data involves 20 neonates videos. Each includes the subject behaviors during daily condition monitoring. All events are performed for each subject and notated by the clinical during condition monitoring. From each video we select the set of events which includes normal health event (pre-disease), disease event, and post-disease event. A total of 60 clips were extracted ranging from $\mathrm{N}$ occurrences of normal event, to $\mathrm{M}$ occurrences of disease event and so on.

We divide the clips into three groups of 20 clips. For instance, each group divided into 2 subset of 10 clips each. In the training phase, we use half of the group and the rest was used for the test task. We consider a recognition task for three class problems including the normal event, disease event and post-disease event respectively. Furthermore, two features descriptors are used in the experiments. The first one is the descriptor of raw thermal signal, we denote it by NRTLBP and the second one is the descriptor based on the approximation components, and we denote it by WNRTLBP. The number of neighboring samples was set to 4 samples for NRTLBP codes, and the Daubechies wavelet with one level decomposition was used for approximation coefficients extraction. We performed the evaluation of our method using linear SVM.

In all experiments, first subset of each group was used for training and the second subset was used for testing. We started the experiment directly by providing the raw features (Mx, and Min raw features) into classifier. The recognition results are in the form of recognition rate, the results of the first experiment are reported in the table 1. We also run the test by using NRTLBP descriptor based on two types of raw features. The results are shown in table 2 . In order to be able to do a full comparison of methods, WNRTLBP based experiments are performed in the same manner as above. Results from this experiment are presented in table 3 . 
Table 1. Recognition rate results, from the Mx and Mn raw features that are directly applied into SVM

\begin{tabular}{|c|c|c|}
\hline Events & $\begin{array}{c}\text { Recognition rate\% } \\
\text { (Mx Temp.value) }\end{array}$ & $\begin{array}{c}\text { Recognition rate\% } \\
\text { (Mn Temp. value) }\end{array}$ \\
\hline Normal Response & 50 & 60 \\
Disease Response & 90 & 60 \\
Post-disease response & 50 & 70 \\
\hline Overall & $63.33 \%$ & $63.33 \%$ \\
\hline
\end{tabular}

Table 2. Recognition rate results, using NRTLBP based raw feature descriptor

\begin{tabular}{|c|c|c|}
\hline Events & $\begin{array}{c}\text { Recognition rate\% } \\
\text { (Mx Temp.value) }\end{array}$ & $\begin{array}{c}\text { Recognition rate\% } \\
\text { (Mn Temp. value) }\end{array}$ \\
\hline Normal Response & 80 & 50 \\
Disease Response & 90 & 90 \\
Post-disease response & 80 & 90 \\
\hline Overall & $83.33 \%$ & $76,67 \%$ \\
\hline
\end{tabular}

Table 3. Recognition rate results, using WNRTLBP based raw feature descriptor

\begin{tabular}{|c|c|c|}
\hline Events & $\begin{array}{c}\text { Recognition rate\% } \\
\text { (Mx Temp.value) }\end{array}$ & $\begin{array}{c}\text { Recognition rate\% } \\
\text { (Mn Temp. value) }\end{array}$ \\
\hline Normal Response & 90 & 100 \\
Disease Response & 100 & 95 \\
Post-disease response & 85 & 93 \\
\hline Overall & $91,667 \%$ & $96 \%$ \\
\hline
\end{tabular}

As we can see, the approach based on the wavelet achieves a good accuracy rate. From a comparison viewpoint, the results that are achieved based on feature descriptors reported that, NRTLBP and WNRTLBP perform best recognition rates when the maximum value of raw feature is used.

\section{Conclusion}

In this paper, we have applied temporal NRLBP as a descriptor of thermal signature from thermal video based on local patch for events recognition. And shown NRTLBP to be suitable for the description of thermal signal containing information about dynamic characteristics of thermal signature. We have extended NRTLBP considering the wavelet transform of feature representation. We have also shown that the power of descriptors for one-dimensional thermal 
signature. The experimental results reveal that non redondant temporal LBP and its modifications tend to be good descriptor based approach for thermal features description.

\section{References}

1. Chekmenev, S.Y., Rara H., and Farag A.A.," Non-contact, wavelet-based measurement of vital signs using thermal imaging'," Graphics Vision Image Proc,2005.

2. Ding, S., Zhu X., Chen W. and Wei D.," Derivation of respiratory signal from singlechannel ECGs based on source statistics," Int. J. Bio. electromagnetism, 6: 43-49, 2004 .

3. Abbas, A. K., Heimann K, jergus K., Orlikowsky T., and leonhardt S.," Neonatal non-contact respiratory monitoring based on real time infrared thermography," Biomedical Engineering online, 2011.

4. Farah AL-Khalidi, Reza Saatchi, Heather Elphick and Derek Burke.," An Evaluation of Thermal Imaging Based Respiration Rate Monitoring in Children," American Journal of Engineering and Applied Sciences 4 (4), pp. 586-597, 2011.

5. Sun N., Garbey M., Merla A. and Pavlidis I.,"Imaging the cardiovascular pulse," In Proceedings of the IEEE Computer Society Conference on Computer Vision and Pattern Recognition, pp. 20-25 2005.

6. Ojala, T., Pietikinen, M., Menp, T.," Multiresolution gray-scale and rotation invariant texture classification with local binary patterns," PAMI ,24 (7), pp. 971987, 2002.

7. Ahonen, T., Hadid, A., Pietikinen, M.," Face recognition with local binary patterns," In: Pajdla, T., Matas, J.G. (eds.) ECCV 2004. LNCS, vol. 3021, pp. 469481. Springer Heidelberg 2004.

8. Ahonen, T., Hadid, A., Pietikinen, M.," Face Description with Local Binary Patterns: Application to Face Recognition," IEEE Transactions on Pattern Analysis and Machine Intelli gence, 28(12), pp. 203720412006.

9. Mallat S.,A wavelet tour of signal processing, the sparse way, 2end Edition, 2001.

10. Doubechies I.,"Orthonormal Bases of Compactly Supported Wavelets," Comm. Pure Applied Math, Vol. 411988. 